\title{
Pathology of Non-Alcoholic Fatty Liver Disease
}

Keywords: Histopathology; Metabolic syndrome; Steatohepatitis; Steatosis

Received: December 01, 2015; Accepted: January 12 2016; Published: January 21, 2016

\section{Introduction}

Due to pandemic of over-nutrition and its related metabolic risks including central obesity, glucose intolerance, dyslipidaemia and hypertension, non-alcoholic fatty liver disease (NAFLD) becomes an alarming global public health issue. NAFLD is the most common metabolic liver disease in the world with prevalence of $10-45 \%$ in different countries [1, 2]. The prevalence of NAFLD is not significantly different between Western countries, [3] and most Asian countries under the influence of "westernized" sedentary lifestyle. NAFLD leads to substantial morbidity and mortality associated with cirrhosis, hepatocellular carcinoma and cardiovascular disease, and becomes the most rapidly growing indication for liver transplantation [2]. Accurate diagnosis of NAFLD is important to facilitate timely and proper management of patients to minimize morbidity and mortality. NAFLD is composed of a full spectrum of conditions from steatosis to non-alcoholic steatohepatitis (NASH) and cirrhosis. Different non-invasive tests, based on clinical, laboratory and radiological tests, have been developed to assess the degree of steatosis, steatohepatitis and fibrosis in NAFLD [2, 4, 5]. Although liver biopsy is an invasive procedure associated with uncommon but severe complications and limited by sampling error, it remains the gold standard for evaluating hepatic pathology in patients with NAFLD, and is recommended in patients with NAFLD at highrisk of steatohepatitis and advanced fibrosis [bridging fibrosis and cirrhosis], and concurrent chronic liver disease of other aetiology [6]. Understanding pathological terminologies of NAFLD is not only important for pathologists in daily diagnostic practice but also essential for hepatologists in communication with pathologists and patients. One should keep in mind that steatosis and even steatohepatitis are not only exceptional to NAFLD and alcoholic liver disease (ALD) but also found in viral hepatitis $C$, drug-induced liver injury (e.g. methotrexate, tamoxifen, steroid), Wilson disease and various metabolic liver diseases. This review article provides a brief overview of fundamental pathological changes of NAFLD and practical tips for general pathologists.

\section{Pathological patterns of NAFLD}

Many essential data on the natural history, clinical characteristics,

\section{Lei Wang ${ }^{1,2}$, Shuangni Y ${ }^{3}$ and Anthony WH Chan ${ }^{4}$}

\author{
1 Department of Pathology, Fudan \\ University Shanghai Cancer Center, \\ Shanghai, China \\ 2 Department of Oncology, Shanghai \\ Medical College, Fudan University, \\ Shanghai, China \\ 3 Department of Pathology, Peking Union \\ Medical College Hospital, Beijing, China \\ 4 Department of Anatomical and Cellular \\ Pathology, State Key Laboratory in \\ Oncology in South China, Prince of \\ Wales Hospital, The Chinese University \\ of Hong Kong
}

Corresponding author: Anthony WH Chan

Đawh_chan@cuhk.edu.hk

Associate Professor, Department of Anatomical and Cellular Pathology, Prince of Wales Hospital, The Chinese University of Hong Kong, Hong Kong.

Tel: 852 2632-2352

Fax: 852 2637-4858

Citation: Wang L, Shuangni Yu, Chan AWH, et al. Pathology of Non-Alcoholic Fatty Liver Disease. Int J Dig Dis. 2016, 2:1.

management and pathological features of NAFLD have been provided by Non-Alcoholic Steatohepatitis Clinical Research Network [NASH-CRN]. NASH-CRN has proposed several histological patterns of NAFLD, which have been already extensively applied in clinical and research settings [7] (Figure 1).

\section{Steatosis with or without inflammation}

Hepatic steatosis (fatty change) is the core pathological change of NAFLD and represents the cytoplasmic accumulation of fat droplets, mainly triglyceride, in hepatocytes. The amount of $5 \%$ is used as the cut-off differentiating between physiological and pathological steatosis according to studies by lipid content measurement and imaging [8]. Macrovesicular and microvesicular steatosis are two morphological forms of hepatic steatosis. Macrovesicular steatosis is traditionally described as a hepatocyte containing a single large fat droplet pushing the nucleus to the periphery (Figure 2A). However, it is not unusual to observe hepatocytes with multiple small to medium-sized fat droplets (Figure 2B) in vicinity to those hepatocytes with a single 


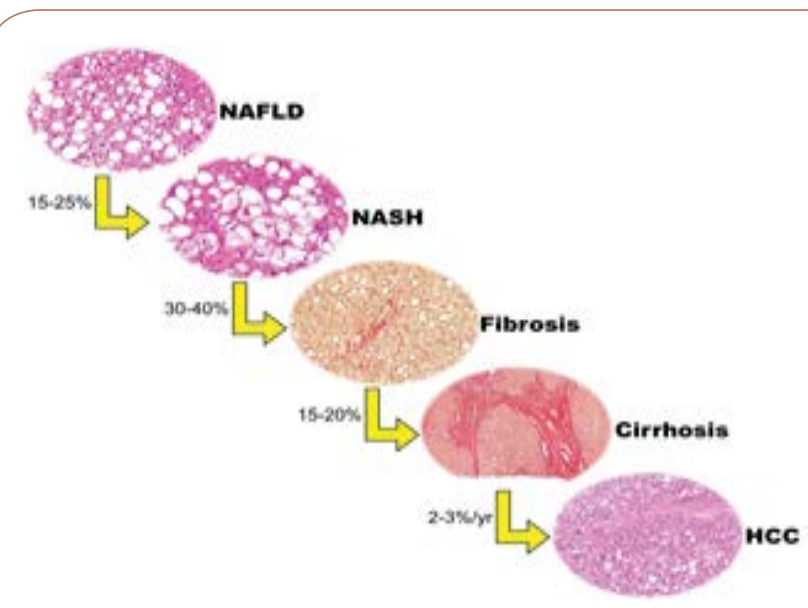

Figure 1 Spectrum of non-alcoholic fatty liver disease.

large fat droplet. A single large fat droplet is believed to be formed by the fusion of multiple small to medium-sized fat droplets [9]. Hence, the term "macrovesicular steatosis" should be broadened to include those hepatocytes with small to medium-sized fat droplets. On the other hand, microvesicular steatosis is featured by the accumulation of much smaller uniform minute fat droplets dispersed throughout the hepatocytes. The hepatocyte with microvesicular steatosis has centrally located nucleus and foamy cytoplasm. Diffuse microvesicular steatosis is typically observed in potentially life-threatening clinical conditions associated with hepatic encephalopathy and acute liver failure secondary to the underlying mitochondrial dysfunction and fatty acid oxidation defect. Reye syndrome, acute fatty liver of pregnancy and acute alcoholic foamy degeneration are classical examples of diffuse microvesicular steatosis. Diffuse microvesicular steatosis is not a pathological feature of NAFLD, whereas focal microvesicular steatosis could be found in $10 \%$ of liver biopsies in patients with NAFLD. The presence of focal microvesicular steatosis in background of conventional macrovesicular steatosis in NAFLD is associated with higher disease severity: higher grades of steatosis, hepatocellular ballooning, inflammation and advanced fibrosis [10].

The degree and distribution of steatosis in a liver biopsy should be recorded in the pathological report. Low power histological examination [i.e., at most $10 x$ and usually $4 x$ objective] is sufficient for evaluation. Assessment of steatosis at higher power should be avoided because the severity of steatosis can be overvalued. The degree of steatosis is semi-quantitatively classified in to 3 grades: mild [5 to $33 \%$ ], moderate (>33 to $66 \%$ ) and marked (>66\%) [11]. The degree of steatosis is correlated with lobular inflammation and centrizonal fibrosis and not associated with hepatocellular ballooning, Mallory-Denk bodies or portal/advanced fibrosis [12]. Predominant zonal distribution of steatosis should be also commented unless steatosis is too mild or the biopsy is too fragmented. There are 4 different patterns of zonal distribution: zone 3 (centrizonal), zone 1 (periportal), panacinar periacinar and azonal. Zone 3 and panacinar distribution patterns are usual patterns in adult NAFLD. Predominant zone 1 distribution is rare in adult patients [1\%] but more typically in paediatric patients [12\%] [11]. Azonal distribution is more commonly found in more severe disease with hepatocellular ballooning, Mallory-Denk bodies and advanced fibrosis [12].
A diagnostic label of "steatosis with inflammation" can be given in the presence of steatosis with lobular and/or portal inflammation. By definition, steatosis with inflammation is not equivalent to steatohepatitis [7]. The inflammatory cells are usually composed of lymphocytes, mononuclear cells and occasionally eosinophils. Compared with ALD, neutrophilic infiltrate is uncommon in NAFLD. Lobular inflammation is featured by small aggregates of macrophages [microgranuloma] or lymphocytes, resembling spotty necrosis in chronic viral hepatitis (Figure 2C). In $80 \%$ of NAFLD patients, the degree of lobular inflammation is usually mild [i.e., <2 foci/20x] [10]. Portal inflammation, which is a typical pathological feature in chronic viral hepatitis, is usually absent or mild in NAFLD (76\% and $77 \%$ in adults and children, respectively) [13]. "More than mild" portal inflammation is defined when at least one portal area shows a moderate to marked density of inflammation and/ or the presence of lymphoid aggregates. Although its presence is associated with steatohepatitis and advanced fibrosis [13], one should consider other causes of chronic hepatitis, particularly viral hepatitis $\mathrm{C}$ which is characterized by moderate/marked portal lymphocytic inflammation with lymphoid follicles and mild steatosis. Predominant portal inflammation exceeding lobular inflammation is more common in paediatric patients [14].

Simple steatosis has been also applied to steatosis with or without inflammation. Simple steatosis is believed to be a benign and non-progressive condition with long-term survival similar to the general population, while steatohepatitis is associated with increased liver-related mortality $[15,16]$. However, Wong et al. showed that $58 \%$ and $28 \%$ of patients with simple steatosis had increased disease activity and fibrosis progression in follow-up biopsies in a 3-year interval, respectively [17]. Moreover, hepatic steatosis could induce oxidative fat injury, endoplasmic reticulum dysfunction and abnormalities of the cytoskeleton resulting in hepatocellular ballooning, which is the characteristic feature of steatohepatitis [18]. Although larger studies are still required to clarify that simple steatosis is potentially progressive lesion [19], one should be reminded that simple steatosis is not always quiescent.

\section{Steatohepatitis}

Steatohepatitis is a characteristic pathological pattern featured by steatosis more than $5 \%$, inflammation and hepatocellular ballooning. Hepatocellular ballooning is the hallmark to distinguish steatohepatitis from steatosis with inflammation, and is characterized by cellular swelling, rarefaction of the hepatocytic cytoplasm and clumped strands of intermediate filaments (Figure 2D). Substantial accumulation of fat droplets, dilatation of the endoplasmic reticulum and cytoskeletal injury contribute to the formation of ballooned hepatocytes [18].

In the early stage of steatohepatitis, ballooned hepatocytes predominantly found in the centrizonal region. Centrizonal distribution of hepatocellular ballooning disappears in later stage or very active steatohepatitis. Mallory-Denk bodies, as known as Mallory bodies and Mallory hyalines are often found in ballooned hepatocytes. They are deeply eosinophilic, ropey intracytoplasmic inclusions (Figure 2D), and represent misfolded protein aggregates composed of primary ubiquitinated cytokeratin $8 / 18$ [CK8/18] and sequestosome 1/p62 [20]. High fat diet contributes to Mallory-Denk bodies through CK8/18 accumulation, CK8 
hyperphosphorylation with subsequent transglutaminase 2-mediated CK18 crosslinking [21]. Although identification of ballooned hepatocytes is crucial in establishing the diagnosis of steatohepatitis, there are significant interobserver (kappa 0.52-0.56 and 0.22 for adult and paediatric cases, respectively) and intraobserver (kappa 0.62-0.66) variabilities in recognizing hepatocellular ballooning $[11,22,23]$. Ballooned hepatocytes should have a clear, not steatotic, cytoplasm with loss of sharp angles regardless of cell size. By using immunohistochemical stain of CK8/18, ballooned hepatocytes show characteristic loss of cytoplasmic expression of $\mathrm{CK} 8 / 18$, whereas residual immunoreactivity is confined to their Mallory-Denk bodies if present (Figure 2E) [24]. However, the identification of hepatocellular ballooning is still relied on conventional $\mathrm{H} \& \mathrm{E}$ section and immunohistochemistry may serve as an ancillary tool in equivocal cases. Although this characteristic CK8/18 is useful to differentiate ballooned hepatocytes in steatohepatitis from hydropic hepatocytes in acute hepatitis, autoimmune hepatitis and chronic viral hepatitis, it is not entirely unique to NASH and ALD and can be shown among hepatocytes with feathery degeneration in chronic cholestatic diseases [24].

\section{Fibrosis}

Fibrosis is a histological parameter signifying chronicity and disease progression. Although it is not one of the diagnostic criteria of steatohepatitis, it is commonly observed in $>80 \%$ patients with NASH irrespective of age [11]. Centrizonal fibrosis and pericellular/perisinusoidal fibrosis (Figure 2F) are the characteristic patterns of fibrosis in fatty liver disease, and represents deposition of fibrous tissue in the space of Disse associated with activation of stellate cells. They are typical in early stage of fibrosis in adult NAFLD/NASH, similar to that in ALD. The fibres tend to be more slender and less marked in NAFLD/NASH than ALD. Periportal fibrosis and bridging fibrosis will be developed as the disease progresses. Eventually, cirrhosis will be established after repetitive hepatic injury, fibrosis, parenchymal extinction and hepatocellular regeneration. In order to properly assess the fibrosis, a good quality connective tissue stain is essential. Common connective tissue stains used in hepatopathology include Masson trichrome, Gordon-Sweets reticulin and Sirius red stains. A good trichrome stain requires an adequate step of differentiation, usually by phosphomolybdic acid. Inadequate or excessive differentiation leads to over- or understaining, which may lead to over- or underestimation of the degree of fibrosis. Sirius red stain is recommended for morphometric quantitation of fibrosis because it provides highly detailed and contrasted staining and is more sensitive in identifying mild perisinusoidal fibrosis [25]. Collagen proportional area [CPA] determined by computer-assisted image analysis could better quantify fibrosis than histological stage, [26] and Sirius red staining for CPA determination was more accurate and reliable for quantifying fibrosis compared with trichrome staining [25]. One pitfall in pathological assessment of fibrosis should be highlighted. The centrizonal fibrotic scar contains aberrant arterioles in about $40 \%$ of patients with $\mathrm{NASH}$, and $55 \%$ of these abnormally arterialized centrizonal scar contains ductular reaction [27]. Pathologists may potentially misinterpret these arterialized centrizonal regions with ductular reaction as portal tracts, resulting in either missed diagnosis or inaccurate staging of NAFLD/NASH. To prevent this misinterpretation, accurate appreciation of normal liver histology is essential. In a normal portal tract, a hepatic artery is usually ( $>90 \%$ ) accompanied by a nearby (within a distance two to three times that of its diameter) interlobular bile duct of similar diameter [28]. Portal tracts have more organized and uniform collagen and elastin deposition than centrizonal scar regions, and well-demarcated limiting plates separating from periportal hepatocytes. However, arterialized centrizonal fibrous scars do not contain any portal vein, or those arterioles/ductule structures are embedded adjacent to or among hepatocytes without separation from the limiting plate [27].

\section{Borderline steatohepatitis}

Definite steatohepatitis is applied for cases fulfilling all diagnostic features of steatohepatitis typically with a predominantly centrizonal distribution. Borderline steatohepatitis is reserved for those cases immediate between steatosis with/without inflammation and definite steatohepatitis. NAFLD-CRN has mentioned 2 forms of borderline steatohepatitis. The first one is zone 3 borderline steatohepatitis and used for those do not have full-blown unequivocal histological features of definite steatohepatitis, including those cases with characteristic centrizonal/ perisinusoidal fibrosis in absence of hepatocellular ballooning, and those cases with equivocal hepatocellular ballooning. The second one is zone 1 borderline steatohepatitis featured by portal-based injury (periportal steatosis, predominantly portal inflammation and portal fibrosis) [11]. Hepatocellular ballooning is usually absent or minimal. This distinctive form of borderline steatohepatitis is a characteristic histological pattern preferentially found in paediatric patients with NASH [75\%]. It is also sometimes referred as type $2 \mathrm{NASH}$ [in contrast to "type 1" NASH in adult] or paediatric NASH in the literature. Boys, younger age, and Asian and Hispanic ethnicity are factors more commonly associated with zone 1 borderline steatohepatitis [14].

\section{Cryptogenic cirrhosis}

Cryptogenic cirrhosis is established after exclusion of viral hepatitis, metabolic, autoimmune and cholestatic liver diseases after an extensive evaluation. It is a common indication for liver transplantation and accounts for $7-14 \%$ of patients requiring liver transplantation. NAFLD is one of leading causes of cryptogenic cirrhosis [29]. The prevalence of diabetes mellitus and obesity in patients with cryptogenic cirrhosis is similar to that of patients with NAFLD and far exceeds that of patients with cirrhosis associated with chronic viral hepatitis and autoimmune hepatitis [30]. Steatosis and/or necroinflammatory activity may resolve or "burn out" as disease progresses to advanced fibrosis in patients with NAFLD/NASH. Careful searching for residual hepatocellular hepatocellular ballooning, Mallory-Denk bodies and perisinusoidal fibrosis, as well as clinical correlation with underlying metabolic risks, are helpful to establish a diagnosis of "burnt-out" NAFLD in cryptogenic cirrhosis [31].

\section{Other pathological lesions in NAFLD}

Some pathological changes that are used to classify the pattern of the disease are briefly mentioned here. Lipogranuloma is composed of a loose aggregate of lymphocytes and histiocytes surrounding a fat globule (Figure 2G), and can be found in NAFLD, ALD and ingestion of mineral oil in food and medication. Glycogenated nuclei represent the nuclear accumulation of 
glycogen (Figure $\mathbf{2 H}$ ), and are observed more commonly in NAFLD than ALD. Although they are probably resulted from impaired glucose tolerance or insulin resistance, they are not pathognomic for NAFLD. They may occur normally in children and young adults ( $11 \%$ and $4 \%$ in the 20 s and early 30 s, respectively) [32], and in other liver diseases including glycogen storage disease, Wilson disease, and other copper overload disorders. Giant mitochondria, as known as megamitochondria, are eosinophilic globular or needle-shaped intracytoplasmic inclusions larger than the nucleus of hepatocytes. Although they are usually found in alcoholic and non-alcoholic fatty liver diseases [33], they may be occasionally observed in different physiologic and pathologic conditions including aging, acute fatty liver of pregnancy, glycogen storage disease and urea cycle defects.

Metabolic syndrome is a significant risk factor for hepatocellular carcinoma (HCC; odds ratio 2.13; 95\% Cl: 1.96-2.31) and intrahepatic cholangiocarcinoma (odds ratio $1.56 ; 95 \% \mathrm{Cl}$ : 1.32-1.83) [34]. Patients with NAFLD-related cirrhosis have an increased risk of developing HCC with incidence of 2-3\% per year [35]. A recently described histological variant of HCC, steatohepatitic HCC, is characterized by HCC exhibiting features of steatohepatitis [steatosis in more than $5 \%$ of tumour cells, hepatocellular ballooning, Mallory-Denk bodies, intratumoral inflammatory infiltrate and perisinusoidal fibrosis], and associated with underlying NAFLD and metabolic risks [36, 37] (Figure 2I).

\section{Pathological grading, staging and scoring systems}

Grading is measure of disease activity and staging is an indicator of disease chronicity. Grading and staging systems have been used in chronic viral hepatitis for decades to provide semiquantification assessment of severity and progression of chronic
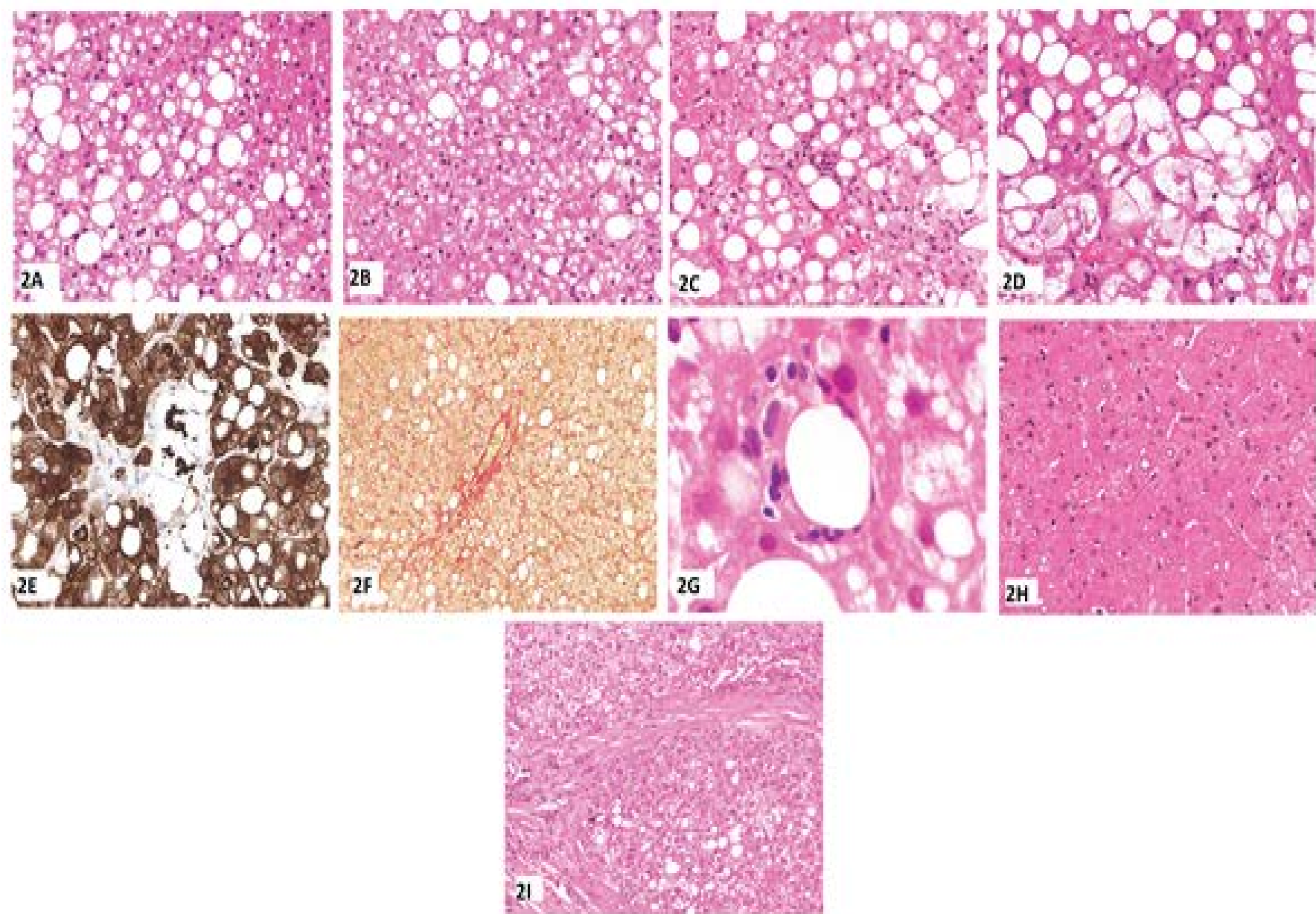

Figure 2 [A] Macrovesicular steatosis: Hepatocytes with predominantly a single large-sized fat droplet. [B] Macrovesicular steatosis: Hepatocytes with multiple small to medium-sized fat droplets. [C] Spotty necrosis: A small clusters of small lymphocytes and histiocytes replace hepatocytes. [D] Hepatocellular ballooning: Swollen hepatocytes exhibit rarefaction of the hepatocytic cytoplasm and clumped strands of intermediate filaments. Some clumped strands of intermediate filaments are qualified as Mallory-Denk bodies. [E] Characteristic loss of cytoplasmic expression in ballooned hepatocytes, whereas residual immunoreactivity is confined to their Mallory-Denk bodies [Immunohistochemistry of cytokeratin 8/18]. [F] Centrizonal and perisinusoidal fibrosis [Sirius red stain]. [G] Lipogranuloma. [H] Glycogenated nuclei. [I] Steatohepatitic hepatocellular carcinoma. 
viral hepatitis [38], and helping in preparing clinical guideline, standardizing pathology reporting and facilitating research studies. The first grading and staging system for NAFLD was proposed by Brunt et al. in 1999. It was derived on liver biopsies from 51 patients with NAFLD. The disease activity grade (0-3) was based by a constellation of histological features including steatosis, lobular and portal inflammation, and hepatocellular ballooning. The fibrosis stage (0-4) was assessed according to fibrosis patterns of adult NAFLD from centrizonal/perisinusoidal to periportal, bridging and cirrhosis [39]. Six years later, NASHCRN released a revised Brunt's system or NASH-CRN system in 2005 [11]. The disease activity grade, known as NAFLD Activity Score (NAS), was the unweighted sum of scores (0-8) for steatosis (0-3), hepatocellular ballooning (0-2), and lobular inflammation $(0-3)$. In the fibrosis staging, early disease (stage 1 ) was further divided into 1a (mild perisinusoidal fibrosis visualized by connective tissue stain only), $1 \mathrm{~b}$ (moderate perisinusoidal fibrosis visualized by H\&E section) and 1c (portal/periportal fibrosis only). In a validation study of the NASH-CRN system in 976 patients, cases with NAS of 0 to 2 were largely considered not diagnostic of definite steatohepatitis (99\%: simple steatosis $75 \%$ and borderline steatohepatitis 24\%), whereas most cases with scores of 5 or more were diagnosed as definite steatohepatitis (86\%) [40]. Cases with NAS of 3 and 4 were distributed almost evenly between all three patterns: steatosis (27\%), borderline steatohepatitis (32\%) and definite steatohepatitis (41\%). One should be reminded that NAS should not be used as the diagnostic criteria for steatohepatitis (i.e., diagnosis of steatohepatitis only if NAS is 5 or more), although an NAS value of 5 or more has been employed as an inclusion criteria of clinical trials treating patients with NASH $[11,40]$. In 2012, Bedossa et al. introduced an algorithm and a scoring system based on a cohort of 679 obese patients receiving bariatric surgery [41]. The FLIP [fatty liver inhibition of progression] algorithm is proposed for segregating lesions into normal liver, NAFLD or NASH by semiquantitative evaluation of steatosis, hepatocellular ballooning, and lobular inflammation. After using this algorithm, the agreement of the diagnosis [NAFLD vs. NASH] increased among expert hepatopathologists from moderate (kappa 0.54) to substantial [kappa 0.66], and much more significantly from fair (kappa 0.35) to substantial (kappa 0.61) among general pathologists received training in liver pathology [42]. The SAF [steatosis, activity, fibrosis] score is the combination of scores of steatosis, activity [hepatocellular ballooning and lobular inflammation] and fibrosis. Compared to the NASH-CRN system, steatosis is no longer a part of the activity score because the prognostic significance of steatosis in disease progression remains controversial. The clinical application of FLIP/SAF system requires further investigations.

\section{Limitations of liver biopsy}

Although liver biopsy is the gold standard diagnostic tool for NAFLD/NASH, it has a few limitations. First, it is an invasive procedure with the incidence of serious complications and mortality of $0.57 \%$ and $<0.01 \%$, respectively $[43,44]$. Transient discomfort at the biopsy site, pain requiring analgesic, vasovagal attack, and transient mild hypotension are common minor complications. Severe complications include haemoperitoneum, pneumothorax, haemothroax, punctures of other organs and biliary peritonitis. The frequency of complications increases with the number of passes performed, and reduces in experienced hands and under imaging guidance [43]. Second, liver biopsy only samples a small proportion (0.001-0.002\%) of the entire liver, and hence sampling error is another limitation. In studies using simultaneous paired biopsies, there were substantial to high agreement (kappa 0.64-0.88) for steatosis grade, fair to high agreement (kappa 0.45-0.87) for hepatocellular ballooning, fair to good agreement (kappa 0.43-0.65) for centrizonal/ perisinusoidal fibrosis, and fair agreement (kappa 0.47) for the overall staging $[22,23]$. Last but not least, intraobserver and interobserver variability is a substantial problem. The consistency in evaluation of steatosis and fibrosis is good to high among different pathologists (kappa 0.64-0.79 and 0.60-0.84, respectively) and for the same pathologist (kappa $0.74-0.83$ and $0.69-0.85$, respectively), while the consistency in assessment of hepatocellular ballooning and lobular inflammation is only fair to good among different pathologists [kappa 0.52-0.56 and 0.330.45 , respectively) and for the same pathologist (kappa 0.62-0.66 and $0.37-0.60$, respectively) $[11,22,23]$.

\section{Conclusion}

Understanding different pathological patterns of NAFLD is important to establish an accurate diagnosis. Grading and staging systems are valuable tools to providing a standard reference in pathology reporting, monitoring disease progression and therapeutic response in daily practice and clinical trials. We should be reminded that the pathological diagnosis of NAFLD/NASH should be relied on interpreting a constellation of histological findings and patterns, and could not be simply replaced by numeric scores. Systematic histological evaluation, full consideration of clinical and laboratory parameters, and good communications with hepatologists are crucial for making an accurate diagnosis of NAFLD and all other medical liver diseases. 


\section{References}

1 Farrell GC, Wong VW, Chitturi S (2013) NAFLD in Asia--as common and important as in the West. Nat Rev Gastroenterol Hepatol 10: 307-318.

2 Rinella ME (2015) Nonalcoholic fatty liver disease: a systematic review. JAMA 313: 2263-2273.

3 Vernon G, Baranova A, Younossi ZM (2011) Systematic review: the epidemiology and natural history of non-alcoholic fatty liver disease and non-alcoholic steatohepatitis in adults. Aliment Pharmacol Ther 34: $274-285$

4 Shen J, Chan HL, Wong GL, Choi PC, Chan AW, et al. (2012) Noninvasive diagnosis of non-alcoholic steatohepatitis by combined serum biomarkers. J Hepatol 56: 1363-1370.

5 Wong GL, Chan HL, Choi PC, Chan AW, Lo AO, et al. (2013) Association between anthropometric parameters and measurements of liver stiffness by transient elastography. Clin Gastroenterol Hepatol 11: 295-302.

6 Chalasani N, Younossi Z, Lavine JE, Diehl AM, Brunt EM, et al. (2012) The diagnosis and management of non-alcoholic fatty liver disease: practice Guideline by the American Association for the Study of Liver Diseases, American College of Gastroenterology, and the American Gastroenterological Association. Hepatology 55: 2005-2023.

7 Yeh MM, Brunt EM (2014) Pathological features of fatty liver disease. Gastroenterology 147: 754-764.

8 Cairns SR, Peters TJ (1983) Biochemical analysis of hepatic lipid in alcoholic and diabetic and control subjects. Clin Sci [Lond] 65: 645652.

9 Martin S, Parton RG (2006) Lipid droplets: a unified view of a dynamic organelle. Nat Rev Mol Cell Biol 7: 373-378.

10 Tandra S, Yeh MM, Brunt EM, Vuppalanchi R, Cummings OW, et al. (2011) Presence and significance of microvesicular steatosis in nonalcoholic fatty liver disease. J Hepatol 55: 654-659.

11 Kleiner DE, Brunt EM, Van Natta M, Behling C, Contos MJ, et al. (2005) Design and validation of a histological scoring system for nonalcoholic fatty liver disease. Hepatology 41: 1313-1321.

12 Chalasani N, Wilson L, Kleiner DE, Cummings OW, Brunt EM, et al. (2008) Relationship of steatosis grade and zonal location to histological features of steatohepatitis in adult patients with nonalcoholic fatty liver disease. J Hepatol 48: 829-834.

13 Brunt EM, Kleiner DE, Wilson LA, Unalp A, Behling CE, et al. (2009) Portal chronic inflammation in nonalcoholic fatty liver disease [NAFLD]: a histologic marker of advanced NAFLD-Clinicopathologic correlations from the nonalcoholic steatohepatitis clinical research network. Hepatology 49: 809-820.

14 Schwimmer JB, Behling C, Newbury R, Deutsch R, Nievergelt C, et al. (2005) Histopathology of pediatric nonalcoholic fatty liver disease. Hepatology 42: 641-649.

15 Ekstedt M, Franzen LE, Mathiesen UL, Thorelius L, Holmqvist M, et al. (2006) Long-term follow-up of patients with NAFLD and elevated liver enzymes. Hepatology 44: 865-873.

16 Rafiq N, Bai C, Fang Y, Srishord M, McCullough A, et al. (2009) Long-term follow-up of patients with nonalcoholic fatty liver. Clin Gastroenterol Hepatol 7: 234-238.

17 Wong VW, Wong GL, Choi PC, Chan AW, Li MK, et al. (2010) Disease progression of non-alcoholic fatty liver disease: a prospective study with paired liver biopsies at 3 years. Gut 59: 969-974.

18 Caldwell S, Ikura Y, Dias D, Isomoto K, Yabu A, et al. (2010) Hepatocellular ballooning in NASH. J Hepatol 53: 719-723.

19 Fielding CM, Angulo P (2014) Hepatic steatosis and steatohepatitis: Are they really two distinct entities? Curr Hepatol Rep 13: 151-158.

20 Strnad P, Harada M, Siegel M, Terkeltaub RA, Graham RM, et al. (2007) Transglutaminase 2 regulates mallory body inclusion formation and injury-associated liver enlargement. Gastroenterology 132: 15151526.

21 Kucukoglu O, Guldiken N, Chen Y, Usachov V, El-Heliebi A, et al. (2014) High-fat diet triggers Mallory-Denk body formation through misfolding and crosslinking of excess keratin 8. Hepatology 60: 169178.

22 Ratziu V, Charlotte F, Heurtier A, Gombert S, Giral P, et al. (2005) Sampling variability of liver biopsy in nonalcoholic fatty liver disease. Gastroenterology 128: 1898-1906.

23 Arun J, Jhala N, Lazenby AJ, Clements R, Abrams GA (2007) Influence of liver biopsy heterogeneity and diagnosis of nonalcoholic steatohepatitis in subjects undergoing gastric bypass. Obes Surg. 17: 155-161.

24 Lackner C, Gogg-Kamerer M, Zatloukal K, Stumptner C, Brunt EM, et al. (2008) Ballooned hepatocytes in steatohepatitis: the value of keratin immunohistochemistry for diagnosis. J Hepatol 48: 821-828.

25 Huang Y, de Boer WB, Adams LA, MacQuillan G, Rossi E, et al. (2013) Image analysis of liver collagen using sirius red is more accurate and correlates better with serum fibrosis markers than trichrome. Liver Int 33: 1249-1256.

26 Calvaruso V, Burroughs AK, Standish R, Manousou P, Grillo F, et al. (2009)Computer-assisted image analysis of liver collagen: relationship to Ishak scoring and hepatic venous pressure gradient. Hepatology 49: 1236-1244.

27 Gill RM, Belt P, Wilson L, Bass NM, Ferrell LD (2011) Centrizonal arteries and microvessels in nonalcoholic steatohepatitis. Am J Surg Pathol 35: 1400-1404.

28 Crawford AR, Lin XZ, Crawford JM (1998) The normal adult human liver biopsy: a quantitative reference standard. Hepatology 28: 323 331.

29 Tardu A, Karagul S, Yagci MA, Ertugrul I, Sumer F, et al. (2015) Histopathological Examination of Explanted Liver After Transplantation in Patients With Cryptogenic Cirrhosis. Transplant Proc 47: 1450-1452.

30 Poonawala A, Nair SP, Thuluvath PJ (2000) Prevalence of obesity and diabetes in patients with cryptogenic cirrhosis: a case-control study. Hepatology 32: 689-692.

31 Caldwell SH, Lee VD, Kleiner DE, Al-Osaimi AM, Argo CK, et al. (2009) NASH and cryptogenic cirrhosis: a histological analysis. Ann Hepatol 8: 346-352.

32 Levene AP, Goldin RD (2010) Physiological hepatic nuclear vacuolation--how long does it persist? Histopathology 56: 426-429.

33 Stewart RV, Dincsoy HP (1982) The significance of giant mitochondria in liver biopsies as observed by light microscopy. Am J Clin Pathol 78: 293-298

34 Welzel TM, Graubard BI, Zeuzem S, El-Serag HB, Davila JA, et al. (2011) Metabolic syndrome increases the risk of primary liver cancer in the 
United States: a study in the SEER-Medicare database. Hepatology 54: 463-471.

35 Ascha MS, Hanouneh IA, Lopez R, Tamimi TA, Feldstein AF, et al. (2010) The incidence and risk factors of hepatocellular carcinoma in patients with nonalcoholic steatohepatitis. Hepatology 51: 19721978.

36 Salomao M, Yu WM, Brown RS, Emond JC, Lefkowitch JH (2010) Steatohepatitic hepatocellular carcinoma [SH-HCC]: a distinctive histological variant of $\mathrm{HCC}$ in hepatitis $\mathrm{C}$ virus-related cirrhosis with associated NAFLD/NASH. Am J Surg Pathol 34: 1630-1636.

37 Shibahara J, Ando S, Sakamoto Y, Kokudo N, Fukayama M (2014) Hepatocellular carcinoma with steatohepatitic features: a clinicopathological study of Japanese patients. Histopathology 64 951-962.

38 Ishak K, Baptista A, Bianchi L, Callea F, De Groote J, et al. (1995) Histological grading and staging of chronic hepatitis. J Hepatol 22 696-699.

39 Brunt EM, Janney CG, Di Bisceglie AM, Neuschwander-Tetri BA, Bacon
BR (1999) Nonalcoholic steatohepatitis: a proposal for grading and staging the histological lesions. Am J Gastroenterol 94: 2467-2474.

40 Brunt EM, Kleiner DE, Wilson LA, Belt P, Neuschwander-Tetri BA, et al. (2011) Nonalcoholic fatty liver disease [NAFLD] activity score and the histopathologic diagnosis in NAFLD: distinct clinicopathologic meanings. Hepatology 53: 810-820.

41 Bedossa P, Poitou C, Veyrie N, Bouillot JL, Basdevant A, et al. (2012) Histopathological algorithm and scoring system for evaluation of liver lesions in morbidly obese patients. Hepatology 56: 1751-1759.

42 Bedossa P, Consortium FP (2014) Utility and appropriateness of the fatty liver inhibition of progression [FLIP] algorithm and steatosis, activity, and fibrosis [SAF] score in the evaluation of biopsies of nonalcoholic fatty liver disease. Hepatology 60: 565-575.

43 Cadranel JF, Rufat P, Degos F (2000) Practices of liver biopsy in France: results of a prospective nationwide survey. For the Group of Epidemiology of the French Association for the Study of the Liver [AFEF]. Hepatology 32: 477-481.

44 Bravo AA, Sheth SG, Chopra S (2001) Liver biopsy. N Engl J Med 344 495-500. 